\title{
Modulation Set Optimization for the Improved Complex Quadrature SM
}

\author{
Asif Iqbal, ${ }^{1}$ Manar Mohaisen $\mathbb{D}^{2},{ }^{2}$ and Kyung Sup Kwak $\mathbb{D}^{1}$ \\ ${ }^{1}$ Department of Information and Communication Engineering, Inha University, Incheon, Republic of Korea \\ ${ }^{2}$ Department of EEC Engineering, KOREATECH, Cheonan, Republic of Korea \\ Correspondence should be addressed to Manar Mohaisen; manar.subhi@koreatech.ac.kr
}

Received 13 February 2018; Revised 4 June 2018; Accepted 6 June 2018; Published 17 July 2018

Academic Editor: Daniele Pinchera

Copyright (c) 2018 Asif Iqbal et al. This is an open access article distributed under the Creative Commons Attribution License, which permits unrestricted use, distribution, and reproduction in any medium, provided the original work is properly cited.

\begin{abstract}
At each channel use, the complex quadrature spatial modulation (CQSM) transmits two signal symbols drawn from two disjoint modulation sets. The indices of the antennas from which symbols are transmitted also carry information. In the improved CQSM (ICQSM), an additional antenna is used to transmit the second signal symbol only when the indices of the antennas to be used for transmission are equal. Conventionally, the second modulation set is a rotated version of the first, where the rotation angle is optimized such that the average unconditional error probability (AUP) is reduced. In this paper, we propose a low-complexity method to design the PSK modulation sets based on reducing the AUP. After introducing min-BER and max- $d_{\min }$, two exhaustive search methods, we analytically show that the AUP depends on Euclidean distance between transmitted vectors, which in turn depends on the power of signal symbols, the Euclidean distance between the symbols of each modulation set, and the Euclidean distance between the symbols of the two sets. The optimal rotation angle is analytically derived for any modulation order and the radii of the modulation sets are optimized such that AUP is reduced for a wide range of system configurations. The simulation results show that more than $3 \mathrm{~dB}$ of power gain is achieved in the case of 16PSK, where higher gains are achieved for higher modulation orders. These gains are achieved at no computational cost because the optimization does not depend on the channel realization.
\end{abstract}

\section{Introduction}

Index modulation (IM) uses the indices of a given resource of the communication system to convey information to the receiver [1]. These indices represent distinct antennas, subcarriers [2], spreading codes [3], or polarities [4]. A multimode orthogonal frequency division multiplexing (OFDM) with IM was proposed in [5], where all the available subcarriers are utilized for transmission and multimodes, that is, signal constellations, are used. IM is also applied to cluster-based wireless sensor networks in [6]. Spatial modulation (SM) is a special case of IM in which information is transmitted not only through signal symbols but also through the index(es) of the antenna(s) from which symbols are transmitted or to which they are designated. This multiple-input multipleoutput (MIMO) gain is achieved while maintaining the transmitter cost as moderate as that of a single-input system $[1,7,8]$. In the conventional spatial modulation (SM), a single signal symbol, drawn from an M-ary phase shiftkeying (PSK) or quadrature amplitude modulation (QAM), is transmitted from a single antenna whose index also carries information [9]. SM is generalized in [10], where a combination of transmit antennas are used to transmit the same signal symbol. The goal of this generalization is to reduce the number of required transmit antennas to achieve a given spectral efficiency. Also, the constraint of the log-two number of transmit antennas imposed in the conventional $\mathrm{SM}$ is relaxed.

A precoding-aided SM (PSM) system was proposed in [11] and generalized in [12]. PSM precodes the single signal symbol using a channel-based precoder such that the symbol is received only by the designated receive antenna whose index carries information. The precoding matrix is generated based on certain performance criterion such as zero-forcing or minimum mean square error [13]. A macrodiversity PSM (MD-PSM) scheme is proposed in [14], where two base 
stations simultaneously communicate with a single user through decentralized precoding.

Quadrature SM (QSM) was proposed in [15], where the spatial constellation was extended to the in-phase and quadrature dimensions. The real part of the single signal symbol is transmitted from the in-phase spatial dimension and the imaginary part from the quadrature spatial dimension. Compared to SM, QSM increases the achieved spectral efficiency by the number of bits transmitted over one spatial dimension. In [16], a differential QSM (DQSM) is proposed to render the recovery of the transmitted information at the receiver possible without the channel knowledge.

In complex QSM (CQSM), two signal symbols are transmitted over the in-phase and quadrature dimensions, leading to further improvement in the spectral efficiency [17]. To alleviate the ambiguity at the receiver, the two signal symbols are drawn from two disjoint modulation sets. When both symbols are transmitted from the same antenna, their sum belongs to the Minkowski sum of the two sets from which the two symbols are drawn. The modulation sets are therefore optimized to minimize the unconditional average bit error rate. The second modulation set is a rotated version of the first. The optimization in [17] reduces to finding the optimal rotation angle that minimizes the average unconditional bit error.

In [18], an improved CQSM (ICQSM) system was proposed, where, compared to CQSM, the transmitter is equipped with an additional antenna. This antenna is used only when the two spatial symbols are equal; that is, both signal symbols are transmitted from the same antenna in CQSM. In this case, the first symbol is transmitted from its designated antenna and the second from the additional antenna. This strategy tremendously reduces the density of the signal symbols in the Euclidean space, leading to a gain of as much as $20 \mathrm{~dB}$ of signal-to-noise ratio (SNR) in the case of 64PSK. Same as in the case of CQSM, the second modulation set is a rotated version of the first.

To reduce the bit error rate (BER), the design of the modulation set or the combination of the number of transmit antennas and the size of modulation set of the SM scheme is of interest. In [19], a bit-to-symbol mapping is proposed assuming channel knowledge at the transmitter, where the performance of the proposed scheme outperforms an earlier scheme proposed in [20]. In [21], the optimization of the number of transmit antennas and the size of the modulation set was conducted with and without the channel knowledge at the transmitter. The effect of the full or partial knowledge of the channel at the transmitter on the design of the spatial modulation schemes was investigated in [22].

The goal of this paper is to design the two modulation sets of the ICQSM to improve the average error performance. We assume that both base stations utilize phase shift keying modulations with the same order [23]. Against the related literature, the contributions of this paper are as follows:

1. We introduce two exhaustive search methods of the optimal/suboptimal modulation sets: (1) minimum BER (min-BER), where the modulation sets are optimized to minimize the instantaneous error rate for a given channel realization, and (2) $\max -d_{\min }$, where the minimum Euclidean distance among the received vectors for a given channel realization is maximized. The first method is optimal and the second is suboptimal. Both methods, as shown below, require high computational complexity and the optimal modulation sets should be updated once the channel changes.

The rest of this paper is organized as follows. In Section 2, the system model and related works are introduced. In Section 3, we introduce the optimal min-BER and the suboptimal max- $d_{\min }$ methods, and propose a low-complexity optimization method that reduces the unconditional average BER. Simulation results and related discussions are given in Section 4. Conclusions are drawn in Section 5.

\section{System Model and Related Works}

2.1. System Model. Consider a MIMO system with $n_{T}$ transmit and $n_{R}$ receive antennas. In the sequel, $n_{T}$ is assumed to be a power of two; hence $N=\log _{2}\left(n_{T}\right)$ is an integer. The received vector is given by

$$
\mathbf{y}=\mathbf{H s}+\mathbf{n},
$$

where $\mathbf{H}$ and $\mathbf{n}$ are the channel matrix and the noise vector whose elements are i.i.d. centered circularly-symmetric complex Gaussian and have a variance of one and $\sigma_{n}^{2}$, respectively, and $\mathbf{s}$ is the transmitted vector.

2.2. Spatial Modulation. In SM, a single symbol $s_{k} \in \Omega$, with $|\Omega|=2^{q}$, is transmitted from the $i$ th transmit antenna, where both $k$ and $i$ carry information and $q$ is the number of bits carried by each signal symbol [9]. The received vector is given by

$$
\mathbf{y}=\mathbf{H e}_{i}^{n_{T}} s_{k}+\mathbf{n}=\mathbf{h}_{i} s_{k}+\mathbf{n},
$$

where $\mathbf{s}=\mathbf{e}_{i}^{n_{T}} s_{k}$ is the transmitted vector and $\mathbf{h}_{i}$ and $\mathbf{e}_{i}^{n}$ are the $i$ th columns of $\mathbf{H}$ and the $n \times n$ identity matrix, respectively. The spectral efficiency of SM is $(q+N)$ bits/s/Hz.

2.3. Quadrature Spatial Modulation. In QSM, at each channel use, a single signal symbol is transmitted and the spatial constellation is extended into in-phase and quadrature dimensions. The real part of the signal symbol is transmitted on the in-phase dimension; the imaginary part is transmitted on the quadrature dimension [15]. Let $\mathfrak{R}\left(s_{k}\right)$ and $\mathfrak{\Im}\left(s_{k}\right)$ be the real and imaginary parts of $s_{k}$; then the received vector is given by

$$
\begin{aligned}
\mathbf{y} & =\mathbf{H s}+\mathbf{n}, \\
& =\mathbf{h}_{i_{1}} \mathfrak{R}\left(s_{k}\right)+j \mathbf{h}_{i_{2}} \mathfrak{I}\left(s_{k}\right)+\mathbf{n},
\end{aligned}
$$

where $k, i_{1}$, and $i_{2}$ carry information. Based on (3), the spectral efficiency of QSM is $(q+2 N)$ bits/s/Hz. 
2.4. Complex Quadrature Spatial Modulation. Instead of transmitting a single signal symbol, CQSM transmits two symbols on the two spatial dimensions [17]. Let $s_{k_{1}} \in \Omega_{a}$ and $s_{k_{2}}^{\prime} \in \Omega_{b}$ be two signal symbols transmitted from the $i_{1}$ th and $i_{2}$ th transmit antennas; then the received vector is given by

$$
\begin{aligned}
\mathbf{y} & =\mathbf{H} \mathbf{s}+\mathbf{n}, \\
& =\mathbf{h}_{i_{1}} s_{k_{1}}+\mathbf{h}_{i_{2}} s_{k_{2}}^{\prime}+\mathbf{n} .
\end{aligned}
$$

Note that $k_{1}, k_{2}, i_{1}$, and $i_{2}$ carry information to the receiver. Therefore, the spectral efficiency of CQSM is $2(q+N)$ bits/s/Hz. Based on (4), we make the following remarks:

1. Since the index of the antenna from which each symbol is transmitted is random, the symbols $s_{k_{1}}$ and $s_{k_{2}}^{\prime}$ should be distinguishable so that the receiver can recover them. This implies that $\Omega_{a}$ and $\Omega_{b}$ should be disjoint.

2. If $i_{1} \neq i_{2}$, one transmitted symbol still belongs to $\Omega_{a}$ and the second belongs to $\Omega_{b}$.

3. If $i_{1}=i_{2}$, the system in (4) reduces to

$$
\mathbf{y}=\mathbf{h}_{i_{1}}\left(s_{k_{1}}+s_{k_{2}}^{\prime}\right)+\mathbf{n}=\mathbf{h}_{i_{1}}\left(s_{k_{3}}^{\prime \prime}\right)+\mathbf{n},
$$

where $s_{k_{3}}^{\prime \prime} \in \Omega_{c}$, with $\Omega_{c}=\Omega_{a} \oplus \Omega_{b}$, and $\oplus$ denotes the Minkowski sum. The size of $\Omega_{c}$ is $\left|\Omega_{c}\right|=\left|\Omega_{a}\right| \times\left|\Omega_{b}\right|$. The full modulation set at the transmitter becomes $\Omega_{d}=\Omega_{a} \cup \Omega_{b} \cup \Omega_{c}$. For instance, if $\Omega_{a}$ and $\Omega_{b}$ are 16PSK sets, $\left|\Omega_{d}\right|=16+16+256=288$. While CQSM outperforms QSM for the same spectral efficiency, the emergence of $\Omega_{c}$ increases the density of symbols in the Euclidean space, leading to a reduction in the Euclidean distance among symbols and hence to a degradation in the error performance.

In CQSM, the set $\Omega_{b}$ is derived from $\Omega_{a}$ as follows:

$$
\Omega_{b}=\left\{s_{k_{2}}^{\prime}=s_{k_{1}} e^{j \theta}, k_{1}=1, \ldots,\left|\Omega_{a}\right|\right\},
$$

where the optimal value of the rotation angle $\theta$ minimizes the bit error rate.

2.5. Improved CQSM. ICQSM is proposed to tremendously reduce the size of $\Omega_{d}$ of CQSM, resulting in an improved error performance. In ICQSM, the transmitter is equipped with an additional antenna that is used to transmit the second signal symbol only when $i_{1}=i_{2}$. Therefore, the received vector is given by

$$
\mathbf{y}=\mathbf{H s}+\mathbf{n}= \begin{cases}s_{k_{1}} \mathbf{h}_{i_{1}}+s_{k_{2}}^{\prime} \mathbf{h}_{i_{2}}+\mathbf{n}, & \text { if } i_{1} \neq i_{2} \\ s_{k_{1}} \mathbf{h}_{i_{1}}+s_{k_{2}}^{\prime} \mathbf{h}_{n_{K}}+\mathbf{n}, & \text { if } i_{1}=i_{2}\end{cases}
$$

where $n_{K}=\left(n_{T}+1\right)$. As a consequence of this strategy, the transmitted vector $\mathbf{s}$ always contains exactly two nonzero elements $s_{k_{1}} \in \Omega_{a}$ and $s_{k_{2}}^{\prime} \in \Omega_{b}$. Therefore, the modulation set at the transmitter becomes $\Omega_{d}=\Omega_{a} \cup \Omega_{b}$. Assuming the base station is using 64PSK modulation sets for both $\Omega_{a}$ and $\Omega_{b},\left|\Omega_{d}\right|=64+64=128$ and $64+64+64 \times 64=$ 4224 symbols in the case of ICQSM and CQSM, respectively. This huge reduction in the size of $\Omega_{d}$ and the density of signal symbols in the Euclidean space increases the Euclidean distance between the signal symbols, leading to an improved bit error performance. For instance, ICQSM outperforms CQSM by more than $20 \mathrm{~dB}$, assuming 64PSK modulation sets [18].

An Example. Let $n_{T}=8, q=2$ and let the message to be transmitted be $\mathbf{m}=\left[\begin{array}{llllllllll}0 & 1 & 0 & 0 & 1 & 1 & 0 & 1 & 1 & 0\end{array}\right]$. The message is split into four parts as follows:

$$
\begin{aligned}
& \mathbf{b}_{1}=\left[\begin{array}{ll}
0 & 1
\end{array}\right], \\
& \mathbf{b}_{2}=\left[\begin{array}{ll}
0 & 0
\end{array}\right], \\
& \mathbf{b}_{3}=\left[\begin{array}{lll}
1 & 1 & 0
\end{array}\right], \\
& \mathbf{b}_{4}=\left[\begin{array}{lll}
1 & 1 & 0
\end{array}\right]
\end{aligned}
$$

where the size of $\mathbf{b}_{1}$ and $\mathbf{b}_{2}$ is equal to $q$ and that of $\mathbf{b}_{3}$ and $\mathbf{b}_{4}$ is equal to $\log _{2}\left(n_{T}\right)$. The bit vectors $\mathbf{b}_{1}$ and $\mathbf{b}_{2}$ modulate the two signal symbols $s_{k_{1}}$ and $s_{k_{2}}^{\prime}$, respectively, where $k_{1}=1$ and $k_{2}{ }^{\prime}=0$. The bit vectors $\mathbf{b}_{3}$ and $\mathbf{b}_{4}$ modulate the indices of the antennas, $i_{1}$ and $i_{2}$, from which $s_{k_{1}}$ and $s_{k_{2}}^{\prime}$ are transmitted, respectively. In this example, $i_{1}=i_{2}=6$.

In the case of CQSM, the transmitted vector is given by

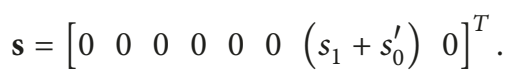

Note that $\mathbf{s}$ includes the single nonzero element $\left(s_{1}+s_{0}^{\prime}\right)$ at the 6th position. The nonzero element in this case belongs to the Minkowski sum of the two sets $\Omega_{a}$ and $\Omega_{b}$, denoted by $\Omega_{c}$, where $s_{k_{1}} \in \Omega_{a}$ and $s_{k_{2}}^{\prime} \in \Omega_{b}$. The size of the Minkowski sum of the two sets is the product of the size of the two sets. This increase in the number of signal symbols in the Euclidean space leads to degradation in error rate.

To overcome this problem, the transmitter in the ICQSM system is equipped with an additional antenna that is used to transmit $s_{k_{2}}^{\prime}$ only when $i_{1}=i_{2}$. Therefore, the transmitted vector is given by

$$
\mathbf{s}=\left[\begin{array}{lllllllll}
0 & 0 & 0 & 0 & 0 & 0 & s_{1} & 0 & s_{0}^{\prime}
\end{array}\right]^{T} .
$$

Accordingly, there are always two active transmit antennas transmitting two signal symbols; the first belongs to $\Omega_{a}$ and the second to $\Omega_{b}$. This reduces the number of signal symbols in the Euclidean space, leading to an improvement in the error rate while keeping the receiver complexity intact. When $i_{1} \neq i_{2}$, the additional antenna is muted and the remaining elements of $\mathbf{s}$ are equivalent for both CQSM and ICQSM systems. It is worth mentioning that ICQSM with an additional antenna outperforms CQSM in terms of the BER, while the latter system uses more antennas in total (the sum of the transmit and receive antennas). 


\section{Proposed Constellation Design}

Let $\mathbf{s}_{i}, \mathbf{s}_{k} \in \mathbb{C}^{n_{K} \times 1}$ be two transmitted vectors, and the corresponding received vectors are $\mathbf{y}_{i}=\mathbf{g}_{i}+\mathbf{n}=\mathbf{H} \mathbf{s}_{i}+\mathbf{n}$ and $\mathbf{y}_{k}=\mathbf{g}_{k}+\mathbf{n}=\mathbf{H} \mathbf{s}_{k}+\mathbf{n}$, respectively. The conditional pairwise error probability (PEP) of the maximum-likelihood receiver is given by $[24,25]$

$$
\begin{aligned}
\operatorname{Pr}\left[\mathbf{g}_{i} \longrightarrow \mathbf{g}_{k} \mid \mathbf{H}\right] & =Q\left(\sqrt{\frac{\left\|\mathbf{y}_{i}-\mathbf{y}_{k}\right\|^{2}}{2 \sigma_{n}^{2}}}\right), \\
& =Q\left(\sqrt{\frac{\left\|\mathbf{H}\left(\mathbf{s}_{i}-\mathbf{s}_{k}\right)\right\|^{2}}{2 \sigma_{n}^{2}}}\right)
\end{aligned}
$$

where

$$
\begin{aligned}
Q(x) & =\frac{1}{\sqrt{(2 \pi)}} \int_{x}^{\infty} e^{-\alpha^{2} / 2} d \alpha \\
& =\frac{1}{\pi} \int_{0}^{\pi / 2} e^{-\alpha^{2} / \sin ^{2}(\alpha)} d \alpha
\end{aligned}
$$

is the Gaussian tail probability function or simply the $Q$ function, and $d_{\mathbf{s}_{i}, \mathbf{s}_{k}}=\left\|\mathbf{s}_{i}-\mathbf{s}_{k}\right\|^{2}$ and $d_{\mathbf{y}_{i}, \mathbf{y}_{k}}=\left\|\mathbf{H}\left(\mathbf{s}_{i}-\mathbf{s}_{k}\right)\right\|^{2}$ are the squared Euclidean distance at the transmitter and the receiver, respectively.

3.1. $\max _{-d_{\min }}$ : Maximizing the Minimum Euclidean Distance. The probability of the conditional error of the ML receiver is approximated by the sum over all possible PEPs, which is given by

$$
P[\mathbf{H}] \leq \frac{1}{2^{M}} \sum_{i=1}^{2^{M}} \sum_{k=1, i \neq k}^{2^{M}} Q\left(\sqrt{\frac{1}{2 \sigma_{n}^{2}} d_{\mathbf{y}_{i}, \mathbf{y}_{k}}}\right),
$$

where $M=2(q+N)$.

At high values of SNR, (13) simplifies to

$$
P[\mathbf{H}] \leq \lambda \cdot Q\left(\sqrt{\frac{1}{2 \sigma_{n}^{2}} d_{\mathbf{y}_{i}, \mathbf{y}_{k}}^{\min }}\right),
$$

where

$$
d_{\mathbf{y}_{i}, \mathbf{y}_{k}}^{\min }=\underset{i, k, i \neq k}{\arg \min }\left\|\mathbf{H}\left(\mathbf{s}_{i}-\mathbf{s}_{k}\right)\right\|^{2},
$$

and $\lambda$ is the number of neighbor points. The Q-function in (14) is a monotonically decreasing function of $d_{\mathbf{y}_{i}, \mathbf{y}_{k}}^{\text {min }}$. Therefore, the error may be decreased by maximizing $d_{\mathbf{y}_{i}, \mathbf{y}_{k}}^{\min }$. The modulation sets are therefore chosen such that

$$
\left(\widehat{\Omega}_{a}, \widehat{\Omega}_{b}\right)=\underset{\Omega_{a}, \Omega_{b}}{\arg \max }\left\{\min _{i, k, i \neq k}\left\|\mathbf{H}\left(\mathbf{s}_{i}-\mathbf{s}_{k}\right)\right\|^{2}\right\} .
$$

The optimization in (16) depends on the instantaneous channel realization $\mathbf{H}$ and the symbol difference $\left(\mathbf{s}_{i}-\mathbf{s}_{k}\right)$. The ICQSM symbol $\mathbf{s} \in \mathbb{C}^{n_{K} \times 1}$ is defined as follows:

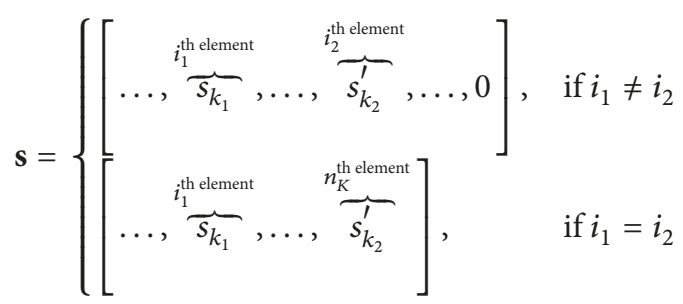

Therefore, for given $\mathbf{H}$ and modulation sets $\Omega_{a}$ and $\Omega_{b}$, the computation of $\mathbf{H}\left(\mathbf{s}_{i}-\mathbf{s}_{k}\right)$ requires precomputing $\mathbf{H} s_{k_{1}}$ and $\mathbf{H s}_{k_{2}}^{\prime}$. Assuming $\left|\Omega_{a}\right|=\left|\Omega_{b}\right|$, computing these two terms requires $8 \cdot n_{R} \cdot n_{K} \cdot\left|\Omega_{a}\right|$ real multiplications. For each pair of symbols $\left(s_{i}, s_{k}\right)$, computing the norm in (16) requires $2 \cdot n_{R}$ real multiplications, leading to a total computational complexity of

$$
\eta^{\text {mul }}=8 \cdot n_{R} \cdot n_{K} \cdot\left|\Omega_{a}\right|+2 \cdot n_{R}\left(2^{M}-1\right) 2^{M}
$$

For instance, for $q=n_{R}=4$ and $n_{K}=5$, evaluating (16) for each pair of $\left(\Omega_{a}, \Omega_{b}\right)$ requires 134,187,520 real multiplications. In this method, only the squared minimum distance of the linear transformation $\mathbf{H}\left(\mathbf{s}_{i}-\mathbf{s}_{k}\right)$ is considered as the search metric. Therefore, even though this method is suboptimal in terms of the BER performance, its computational complexity is very high. Furthermore, this computation should be performed for each channel realization.

3.2. min-BER: Minimizing the Conditional Error Rate. Based on (11) and referring to [26], the upper bound of the conditional BER is formulated as follows:

$$
P_{e}[\mathbf{H}] \leq \frac{1}{M 2^{M}} \sum_{i=1}^{2^{M}} \sum_{k=1, i \neq k}^{2^{M}} D_{\mathbf{s}_{i}, \mathbf{s}_{k}} \cdot Q\left(\sqrt{\frac{1}{2 \sigma_{n}^{2}} d_{\mathbf{y}_{i}, \mathbf{y}_{k}}}\right),
$$

where $D_{\mathbf{s}_{i}, \mathbf{s}_{k}}$ is the Hamming distance between $\mathbf{s}_{i}$ and $\mathbf{s}_{k}$, that is, the number of errors associated with the event $\mathbf{s}_{i} \longrightarrow \mathbf{s}_{k} \mid$ H. The optimal modulation sets are therefore given by

$$
\left(\widehat{\Omega}_{a}, \widehat{\Omega}_{b}\right)=\underset{\Omega_{a}, \Omega_{b}}{\arg \min }\left\{P_{e}[\mathbf{H}]\right\} .
$$

Evaluating (20) for each pair $\left(\Omega_{a}, \Omega_{b}\right)$ requires (1) computing $d_{\mathbf{y}_{i}, \mathbf{y}_{j}}=\left\|\mathbf{H}\left(\mathbf{s}_{i}-\mathbf{s}_{k}\right)\right\|^{2}$, (2) evaluating the $Q$-function $2^{M} \cdot\left(2^{M}-1\right)$ times, and (3) $2^{M} \cdot\left(2^{M}-1\right)$ real multiplications for multiplying the Q-function by $D_{\mathbf{s}_{\mathrm{i}}, \mathbf{s}_{k}}$. The first term is computed in (18). The exact computation of the Qfunction is complex as shown in (12). Alternatively, several approximations of the $Q$-function, which vary in terms of computational complexity and accuracy, were proposed in $[27,28]$. In [28], the Q-function is approximated using a second-order exponential model as follows:

$$
Q(x) \approx e^{a x^{2}+b x+c},
$$

where the real numbers $a, b$, and $c$ are the fitting parameters. Evaluating (21) requires 3 real multiplications 
and an evaluation of the exponential function, which in turn requires $13 \cdot \log _{2}(n)$ real multiplications, with $n$ denoting the number of digits of precision (Theorem 10.1 in [29]). For $n=8,13 \cdot \log _{2}(8)=39$, leading to a total of 42 real multiplications to evaluate (21). This leads to a total computational complexity of

$$
\eta^{\mathrm{mul}}=8 \cdot n_{R} \cdot n_{K} \cdot\left|\Omega_{a}\right|+2^{M} \cdot\left(2^{M}-1\right) \cdot\left(2 n_{R}+43\right) .
$$

The search for the optimal modulation sets depends on the instantaneous channel realization $\mathbf{H}$. Assuming that the optimization is performed over a moderate number of ten combinations $\left(\Omega_{a}, \Omega_{b}\right)$, the search requires ten times the number of real multiplications of (22). This turns the search unfeasible for large $M$ and $n_{R}$.

A method to reduce the computational complexity is explained as follows. The Q-function in (19) is monotonically decreasing; that is, $Q\left(x_{1}\right)<Q\left(x_{2}\right)$ if $x_{1}>x_{2}$. For instance, $Q(10)=7.62 \times 10^{-24}$. This implies that a greedy algorithm can be used to exclude the computation of the $Q$-function for arguments larger than a given threshold. This proposal is of interest at high values of SNR as the denominator of the argument of $Q(\cdot)$ in (19) becomes small. However, the computational complexity of this algorithm is still high.

3.3. Reducing the Unconditional Error Rate. The performance gains achieved through choosing the modulation sets that minimize the receive Euclidean distance $d_{\mathbf{y}_{i}, \mathbf{y}_{k}}$ in (16) or those that minimize the BER in (20) come at a high computational cost. Since both search problems depend on the channel realization $\mathbf{H}$, computations should track the change in the channel and the transmitter should have perfect knowledge of the channel state information. Suboptimal modulation sets are obtained through assuming the unconditional BER as the cost function for the search problem on hand. The unconditional BER is obtained by averaging the upper bound in (19) over $\mathbf{H}$, resulting in

$$
\bar{P}_{e}=\frac{1}{M 2^{M}} \sum_{i=1}^{2^{M}} \sum_{k=1, i \neq k}^{2^{M}} D_{\mathbf{s}_{i}, \mathbf{s}_{k}} \operatorname{Pr}\left[\mathbf{g}_{i} \longrightarrow \mathbf{g}_{k}\right]
$$

where the unconditional PEP is given by:

$$
\operatorname{Pr}\left[\mathbf{g}_{i} \longrightarrow \mathbf{g}_{k}\right]=\gamma_{i, j}^{n_{R}} \sum_{l=0}^{n_{R}-1}\left(\begin{array}{c}
n_{R}-1+l_{l} \\
l
\end{array}\right)\left[1-\gamma_{i, j}\right]^{l}
$$

and

$$
\gamma_{i, j}=\frac{1}{2}\left(1-\sqrt{\frac{\bar{\zeta}_{i, j} / 2}{1+\bar{\zeta}_{i, j} / 2}}\right)
$$

Assuming that the elements of $\mathbf{H}$ are independent and circularly-symmetric Gaussian with mean and variance of zero and one, respectively, $\bar{\zeta}$ is given by

$$
\bar{\zeta}_{i, j}=\mathbb{E}_{\mathbf{H}}\left\{\frac{d_{\mathbf{y}_{i}, \mathbf{y}_{k}}}{2 \sigma_{n}^{2}}\right\}=\frac{d_{\mathbf{s}_{i}, \mathbf{s}_{k}}}{2 \sigma_{n}^{2}}=\frac{\left\|\left(\mathbf{s}_{i}-\mathbf{s}_{k}\right)\right\|^{2}}{2 \sigma_{n}^{2}}
$$

The asymptotic unconditional PEP, obtained by taking the Taylor series of (24) and ignoring the higher order terms, is given by

$$
\begin{aligned}
\operatorname{Pr}\left[\mathbf{g}_{i} \longrightarrow \mathbf{g}_{k}\right] & \approx \frac{2^{n_{R}-1} \Gamma\left(n_{R}+0.5\right)}{\sqrt{\pi}\left(n_{R}\right) !}\left(\frac{2}{\rho \cdot d_{\mathbf{s}_{i}, \mathbf{s}_{k}}}\right)^{n_{R}} \\
& =\left(\begin{array}{c}
2 n_{R}-1 \\
n_{R}
\end{array}\right) \rho^{-n_{R}}\left(d_{\mathbf{s}_{i}, \mathbf{s}_{k}}\right)^{-n_{R}}
\end{aligned}
$$

where $\rho=1 / \sigma_{n}^{2}$ is the signal-to-noise ratio (SNR) [15].

It turns out that, for large values of $\rho$, the unconditional average BER in (27) depends only on $d_{\mathbf{s}_{i}, \mathbf{s}_{k}}$ and $n_{R}$. While the effect of $n_{R}$ on the error rate is evident, the effect of $d_{\mathbf{s}_{i}, \mathbf{s}_{k}}$ requires further explanation. The value of $d_{\mathbf{s}_{i}, \mathbf{s}_{k}}$ depends on the mutual relationship between $i_{1}, i_{2}, \hat{i}_{1}$, and $\hat{i}_{2}$. For these four parameters, there exist 64 relational conditions. Among these conditions, only fifteen are not contradictory, which lead to the following seven different values of $d_{\mathbf{s}_{i}, \mathbf{s}_{k}}$ :

(1) $\left|s_{k_{1}}\right|^{2}+\left|s_{k_{2}}^{\prime}\right|^{2}+\left|\widehat{s}_{k_{1}}\right|^{2}+\left|\widehat{s}_{k_{2}}^{\prime}\right|^{2}$,

if $\left(i_{1} \neq i_{2}, \widehat{i_{1}} \neq \widehat{i_{2}}, i_{1} \neq \widehat{i_{1}}, i_{2} \neq \widehat{i_{2}}, i_{1} \neq \widehat{i_{2}}, i_{2} \neq \widehat{i_{1}}\right)$

or $(\underbrace{i_{1}=i_{2}}_{i_{2}=n_{K}}, \widehat{i_{1}} \neq \widehat{i_{2}}, i_{1} \neq \widehat{i_{1}}, i_{2} \neq \widehat{i_{2}}, i_{1} \neq \widehat{i_{2}}, i_{2} \neq \widehat{i_{1}})$

or $(i_{1} \neq i_{2}, \underbrace{\widehat{i_{1}}=\widehat{i_{2}}}_{\hat{i}_{2}=n_{K}}, i_{1} \neq \widehat{i_{1}}, i_{2} \neq \widehat{i_{2}}, i_{1} \neq \widehat{i_{2}}, i_{2} \neq \widehat{i_{1}})$

(2) $\left|s_{k_{2}}^{\prime}-\widehat{s}_{k_{1}}\right|^{2}+\left|s_{k_{1}}\right|^{2}+\left|\widehat{s}_{k_{2}}^{\prime}\right|^{2}$

if $\left(i_{1} \neq i_{2}, \widehat{i_{1}} \neq \widehat{i}_{2}, i_{1} \neq \hat{i}_{1}, i_{2} \neq \widehat{i}_{2}, i_{1} \neq \widehat{i}_{2}, i_{2}=\widehat{i_{1}}\right)$

if $(i_{1} \neq i_{2}, \underbrace{\widehat{i_{1}}=\hat{i}_{2}}_{\hat{i}_{2}=n_{K}}, i_{1} \neq \widehat{i}_{1}, i_{2}=\widehat{i}_{2}, i_{1} \neq \widehat{i}_{2}, i_{2}=\widehat{i_{1}})$

(3) $\left|s_{k_{1}}-\hat{s}_{k_{2}}^{\prime}\right|^{2}+\left|s_{k_{2}}^{\prime}\right|^{2}+\left|\widehat{s}_{k_{1}}\right|^{2}$

if $\left(i_{1} \neq i_{2}, \widehat{i_{1}} \neq \widehat{i_{2}}, i_{1} \neq \widehat{i_{1}}, i_{2} \neq \widehat{i_{2}}, i_{1}=\widehat{i_{2}}, i_{2} \neq \widehat{i_{1}}\right)$

if $(\underbrace{i_{1}=i_{2}}_{i_{2}=n_{K}}, \widehat{i_{1}} \neq \widehat{i}_{2}, i_{1} \neq \widehat{i}_{1}, i_{2}=\widehat{i}_{2}, i_{1}=\widehat{i}_{2}, i_{2} \neq \widehat{i}_{1})$

(4) $\left|s_{k_{1}}-\widehat{s}_{k_{2}}^{\prime}\right|^{2}+\left|s_{k_{2}}^{\prime}-\widehat{s}_{k_{1}}\right|^{2}$

if $\left(i_{1} \neq i_{2}, \widehat{i_{1}} \neq \widehat{i_{2}}, i_{1} \neq \widehat{i_{1}}, i_{2} \neq \widehat{i_{2}}, i_{1}=\widehat{i_{2}}, i_{2}=\widehat{i_{1}}\right)$

(5) $\left|s_{k_{2}}^{\prime}-\widehat{s}_{k_{2}}^{\prime}\right|^{2}+\left|s_{k_{1}}\right|^{2}+\left|\widehat{s}_{k_{1}}\right|^{2}$

if $\left(i_{1} \neq i_{2}, \widehat{i_{1}} \neq \widehat{i_{2}}, i_{1} \neq \widehat{i_{1}}, i_{2}=\widehat{i_{2}}, i_{1} \neq \widehat{i_{2}}, i_{2} \neq \widehat{i_{1}}\right)$

or $(\underbrace{i_{1}=i_{2}}_{i_{2}=n_{K}}, \underbrace{\widehat{i_{1}}=\widehat{i_{2}}}_{\hat{i}_{2}=n_{K}}, i_{1} \neq \widehat{i_{1}}, i_{2} \neq \widehat{i_{2}}, i_{1} \neq \widehat{i}_{2}, i_{2} \neq \widehat{i_{1}})$ 
TABLE 1: Probability of $d_{\mathbf{s}, \widehat{\mathbf{s}}}^{2}$ for several values of $n_{T}$.

\begin{tabular}{lccccc}
\hline$n_{T}$ & $p_{1}$ & $p_{2}$ & $p_{4}$ & $p_{6}$ & $p_{7}$ \\
\hline 2 & 0 & 0.125 & 0.125 & 0.25 & 0.0 .25 \\
4 & 0.2813 & 0.1406 & 0.1469 & 0.1875 & 0.0625 \\
16 & 0.769 & 0.0549 & 0.0037 & 0.0586 & 0.0039 \\
128 & 0.9691 & 0.0077 & $6.056 \times 10^{-5}$ & 0.0078 & $6.1035 \times 10^{-5}$ \\
\hline
\end{tabular}

(6) $\left|s_{k_{1}}-\widehat{s}_{k_{1}}\right|^{2}+\left|s_{k_{2}}^{\prime}\right|^{2}+\left|\widehat{s}_{k_{2}}^{\prime}\right|^{2}$,

if $\left(i_{1} \neq i_{2}, \hat{i}_{1} \neq \hat{i}_{2}, i_{1}=\hat{i}_{1}, i_{2} \neq \hat{i}_{2}, i_{1} \neq \hat{i}_{2}, i_{2} \neq \widehat{i}_{1}\right)$

or $(\underbrace{i_{1}=i_{2}}_{i_{2}=n_{K}}, \widehat{i_{1}} \neq \hat{i}_{2}, i_{1}=\hat{i}_{1}, i_{2} \neq \widehat{i}_{2}, i_{1} \neq \widehat{i}_{2}, i_{2}=\widehat{i_{1}})$

or $(i_{1} \neq i_{2}, \underbrace{\widehat{i_{1}}=\widehat{i_{2}}}_{\hat{i}_{2}=n_{K}}, i_{1}=\hat{i}_{1}, i_{2} \neq \widehat{i}_{2}, i_{1}=\widehat{i}_{2}, i_{2} \neq \widehat{i_{1}})$

(7) $\left|s_{k_{1}}-\widehat{s}_{k_{1}}\right|^{2}+\left|s_{k_{2}}^{\prime}-\widehat{s}_{k_{2}}^{\prime}\right|^{2}$,

if $\left(i_{1} \neq i_{2}, \widehat{i_{1}} \neq \widehat{i_{2}}, i_{1}=\widehat{i_{1}}, i_{2}=\widehat{i_{2}}, i_{1} \neq \widehat{i_{2}}, i_{2} \neq \widehat{i_{1}}\right)$

or $(\underbrace{i_{1}=i_{2}}_{i_{2}=n_{K}}, \underbrace{\hat{i}_{1}=\widehat{i_{2}}}_{\hat{i}_{2}=n_{K}}, i_{1}=\widehat{i_{1}}, i_{2}=\widehat{i_{2}}, i_{1}=\widehat{i_{2}}, i_{2}=\widehat{i_{1}})$

Let $f_{i}^{k}$ be the frequency of the $k$ th condition leading to the $i$ th value of $d_{\mathbf{s}_{i}, \mathbf{s}_{k}}$. The expressions of these frequencies are given as follows:

$$
\begin{aligned}
& f_{1}^{1}=n_{T}\left(n_{T}-1\right)\left(n_{T}-2\right)\left(n_{T}-3\right) \\
& f_{1}^{2}=f_{1}^{3}=f_{2}^{1}=f_{3}^{1}=f_{5}^{1}=f_{6}^{1}=n_{T}\left(n_{T}-1\right)\left(n_{T}-2\right) \\
& f_{2}^{2}=f_{3}^{2}=f_{4}^{1}=f_{5}^{2}=f_{6}^{2}=f_{6}^{3}=f_{7}^{1}=n_{T}\left(n_{T}-1\right) \\
& f_{7}^{2}=n_{T}
\end{aligned}
$$

Let the probability of the $i$ th value of $d_{\mathbf{s}_{i}, \mathbf{s}_{k}}$, for $i=1, \ldots, 7$, listed in (28) be denoted by $p_{i}=\sum_{k} f_{i}^{k} / n_{T}^{4}$; then

$$
\begin{aligned}
& p_{1}=\frac{\left(n_{T}-1\right)^{2}\left(n_{T}-2\right)}{n_{T}^{3}}, \\
& p_{2}=p_{3}=p_{5}=\frac{\left(n_{T}-1\right)^{2}}{n_{T}^{3}} \\
& p_{4}=\frac{n_{T}-1}{n_{T}^{3}}, \\
& p_{6}=\frac{n_{T}-1}{n_{T}^{2}} \\
& p_{7}=\frac{1}{n_{T}^{2}}
\end{aligned}
$$

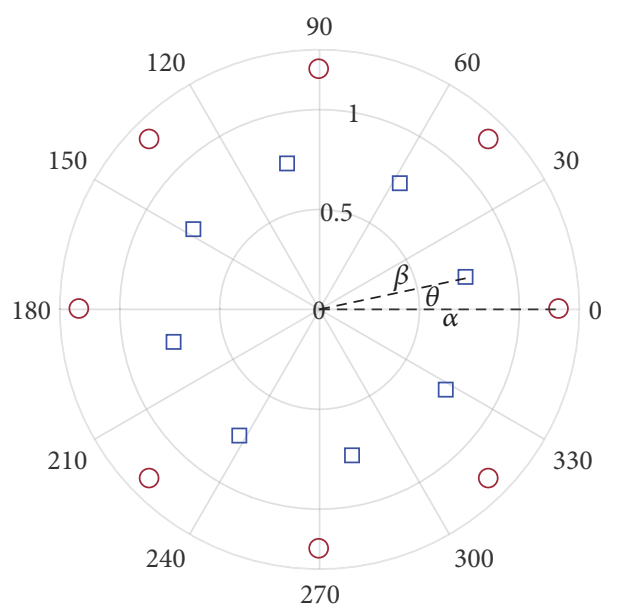

FIGURE 1: An example of the modulation sets $\Omega_{a}$ and $\Omega_{b}$, assuming 8PSK.

As $n_{T}$ becomes large, $p_{1} \longrightarrow 1, p_{2}, p_{3}, p_{5}, p_{6} \longrightarrow 1 / n_{T}$, and $p_{4}, p_{7} \longrightarrow 1 / n_{T}^{2}$. Table 1 lists these probabilities for several values of $n_{T}$. The convergence of these probabilities is very clear as $n_{T}$ takes relatively large values.

As stated earlier, both $\Omega_{a}$ and $\Omega_{b}$ are PSK modulation sets, with different, or equal, radii of $\alpha$ and $\beta$, respectively. In addition to $\alpha$ and $\beta$, the rotation angle $\theta$ is another parameter that affects the value of $d_{\mathbf{s}_{i}, \mathbf{s}_{k}}$. Due to the symmetric structure of the PSK modulation sets, the range over which $\theta$ is optimized is $[0, \phi / 2]$, where $\phi=2 \pi /|\Omega|$ is the angle between any two adjacent symbols in the modulation set $\Omega$. An example using 8PSK modulation sets for both $\Omega_{a}$ and $\Omega_{b}$ is depicted in Figure 1. In light of this discussion, we make the following remarks regarding the design of the constellation sets $\Omega_{a}$ and $\Omega_{b}$, where the goal is to maximize $d_{\mathbf{s}_{i}, \mathbf{s}_{k}}$, leading to an improved average BER, as shown in (27).

(1) The first value of $d_{\mathbf{s}_{i}, \mathbf{s}_{k}}$ in (28) implies that the power sum $\left|s_{k_{1}}\right|^{2}+\left|s_{k_{2}}^{\prime}\right|^{2}$ should be maximized. There is no constraint on the distribution of the total power among the symbols $s_{k_{1}} \in \Omega_{a}$ and $s_{k_{1}}^{\prime} \in \Omega_{b}$. To fulfill the requirement of an average transmission power of one per signal symbol, $\mathbb{E}\left(s_{k_{1}}^{*} s_{k_{1}}\right)+\mathbb{E}\left(s_{k_{2}}^{\prime *} s_{k_{2}}^{\prime}\right)=\alpha^{2}+\beta^{2}=$ 2. Any two conventional PSK modulation sets satisfy this condition. In this case, $d_{\mathbf{s}_{i}, \mathbf{s}_{k}}^{2}$ is fixed and is equal to 4 .

(2) The second and third values of $d_{\mathbf{s}_{i}, \mathbf{s}_{k}}$ in (28) imply that

(i) $d\left(\Omega_{a}, \Omega_{b}\right)=\left|s_{k_{1}}-s_{k_{2}}^{\prime}\right|^{2}$ should be maximized, 
(ii) the power sum $\left|s_{k_{2}}^{\prime}\right|^{2}+\left|s_{k_{1}}\right|^{2}=\alpha^{2}+\beta^{2}$ should be maximized.

Due to the transmission power constraint, $\alpha^{2}+\beta^{2}=2$ is always kept fixed. For each symbol $s_{k_{1}} \in \Omega_{a}, k_{1}=$ $1, \ldots,\left|\Omega_{a}\right|$,

$$
\begin{aligned}
d_{i}\left(\Omega_{a}, \Omega_{b}\right) & =\alpha^{2}+\beta^{2}-2 \alpha \beta \cos (\theta+i \phi), \\
& =2-2 \alpha \beta \cos (\theta+i \phi)
\end{aligned}
$$

for $i=0, \ldots,\left|\Omega_{a}\right|-1$. Regardless of the values of $\alpha, \beta$, and $\theta$ and due to the symmetry of the modulation set, summing the second term of (31), that is, $2 \alpha \beta \cos (\theta+$ $i \phi)$, over all the values of $i$ is equal to zero. Hence the total squared Euclidean distance from any symbol in $\Omega_{a}$ to all the symbols in $\Omega_{b}$ is equal to $2\left|\Omega_{b}\right|$. As such, we choose to maximize the minimum Euclidean distance $d_{\min }\left(\Omega_{a}, \Omega_{b}\right)$.

(3) Based on the fourth value of $d_{s_{i}, s_{k}}$, the following condition should be fulfilled to improve the BER:

(i) The minimum value of the Euclidean distance $d_{\min }\left(\Omega_{a}, \Omega_{b}\right)=\min \left(\left|s_{k_{1}}-\hat{s}_{k_{2}}^{\prime}\right|^{2}\right)$ should be maximized.

Due to the sum of the two Euclidean distances in the fourth value, the resulting $d_{\mathrm{s}_{i}, s_{k}}$ is the Minkowski sum of $\left|s_{k_{1}}-\hat{s}_{k_{2}}^{\prime}\right|^{2}$ to itself. In this case, values will range from $2 \min \left(d_{s_{i}, s_{k}}\right)=4-4 \alpha \beta \cos (\theta)$ to $2 \max \left(d_{s_{i}, s_{k}}\right)=$ $4+4 \alpha \beta \cos (\theta)$.

(4) The fifth value of $d_{s_{i}, s_{k}}$ implies that

(i) the minimum value of the power sum $\left|s_{k_{1}}\right|^{2}+$ $\left|\widehat{s}_{k_{1}}\right|^{2}=2 \alpha^{2}$ should be maximized,

(ii) the minimum Euclidean distance $d_{\min }\left(\Omega_{b}\right)=$ $\min \left(\left|s_{k_{2}}^{\prime}-\hat{s}_{k_{2}}\right|^{2}\right)$ should be maximized,

(1) Optimization of the Rotation Angle. Due to the structure of the PSK modulation sets, the rotation angle $\theta$ takes on values in the interval $[0, \phi / 2]$. Based on the given analysis and (28), the optimization of the rotation angle for given $(\alpha, \beta)$ reduces to

$$
\begin{aligned}
\theta^{*} & =\underset{\theta \in[0, \phi / 2]}{\arg \max }\left\{d_{\min }\left(\Omega_{a}\right), d_{\min }\left(\Omega_{b}\right), d_{\min }\left(\Omega_{a}, \Omega_{b}\right)\right\} \\
& =\underset{\theta \in[0, \phi / 2]}{\arg \max }\left\{d_{\min }\left(\Omega_{a}, \Omega_{b}\right)\right\} \\
& =\underset{\theta \in[0, \phi / 2]}{\arg \max }\{2-2 \alpha \beta \cos (\theta)\} \\
& =\frac{\phi}{2},
\end{aligned}
$$

where $d_{\min }\left(\Omega_{a}\right)$ and $d_{\min }\left(\Omega_{b}\right)$ do not depend on $\theta$. Also, the cosine function is strictly decreasing in $[0, \phi / 2]$, which implies that $\theta^{*}=\phi / 2$. This analytical results coincide with the simulation results in [18].
TABLE 2: Optimal largest radius, $\alpha_{\text {opt }}$, for several system configurations.

(a) $n_{T}=n_{R}=4$ for several modulation schemes

\begin{tabular}{lcc}
\hline SNR $(\mathrm{dB})$ & $q$ & $\alpha_{\text {opt }}$ \\
\hline 13 & 2 & 1 \\
16 & 3 & 1.18 \\
21 & 4 & 1.13 \\
25 & 5 & 1.09 \\
30 & 6 & 1.06 \\
37 & 7 & 1.04 \\
\hline
\end{tabular}

(b) $n_{R}=q=4$ for several values of $n_{T}$

\begin{tabular}{lcc}
\hline SNR $(\mathrm{dB})$ & $n_{T}$ & $\alpha_{\text {opt }}$ \\
\hline 19 & 2 & 1.12 \\
21 & 4 & 1.13 \\
20 & 8 & 1.15 \\
20 & 16 & 1.15 \\
21 & 32 & 1.15 \\
\hline
\end{tabular}

(c) $16 \mathrm{PSK}$ for several values of $n_{T}=n_{R}$

\begin{tabular}{lcc}
\hline SNR $(\mathrm{dB})$ & $n_{T}$ & $\alpha_{\text {opt }}$ \\
\hline 30 & 2 & 1.15 \\
21 & 4 & 1.13 \\
15 & 8 & 1.13 \\
12 & 16 & 1.12 \\
9 & 32 & 1.11 \\
\hline
\end{tabular}

(d) $n_{T}=q=4$ for several values of $n_{R}$

\begin{tabular}{lcc}
\hline SNR $(\mathrm{dB})$ & $n_{R}$ & $\alpha_{\text {opt }}$ \\
\hline 28 & 2 & 1.16 \\
21 & 4 & 1.13 \\
15 & 8 & 1.12 \\
12 & 16 & 1.12 \\
8 & 32 & 1.12 \\
\hline
\end{tabular}

(2) Optimization of the Modulation Sets' Radii. Based on (28) and the related discussion, the optimal radii should jointly maximize the following cost functions:

$$
\begin{gathered}
\left(\alpha^{*}, \beta^{*}\right)=\underset{\alpha, \beta}{\arg \max }\left\{\min \left(\left|s_{k_{1}}\right|^{2}\right), \min \left(\left|s_{k_{2}}^{\prime}\right|^{2}\right),\right. \\
\left.d_{\min }\left(\Omega_{a}\right), d_{\min }\left(\Omega_{b}\right), d_{\min }\left(\Omega_{a}, \Omega_{b}\right)\right\} .
\end{gathered}
$$

The joint optimization of (33) is hard to achieve because several conditions are contradictory and the optimization process is solved over a large, continuum range of values for several parameters. For instance, when $\beta$ increases, $\alpha$ decreases and $d_{\min }\left(\Omega_{a}\right)$ will decrease, and so on.

Based on the above discussion, the optimal value of $\alpha$ which minimizes the average BER is obtained through simulations. The simulation results for several scenarios are depicted in Figure 2 and $\alpha_{\text {opt }}$ is listed in Table 2. For each case, the minimum BER value is indicated by a black asterisk 


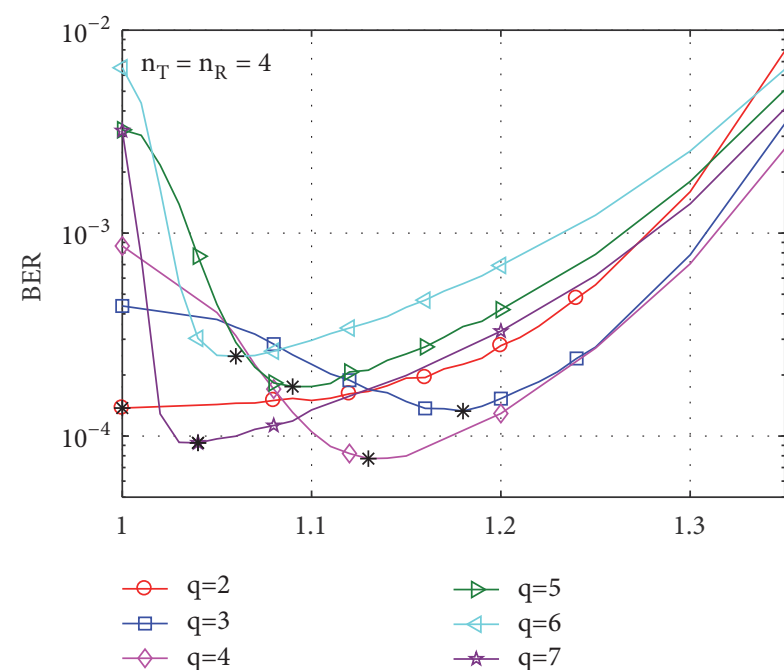

(a)

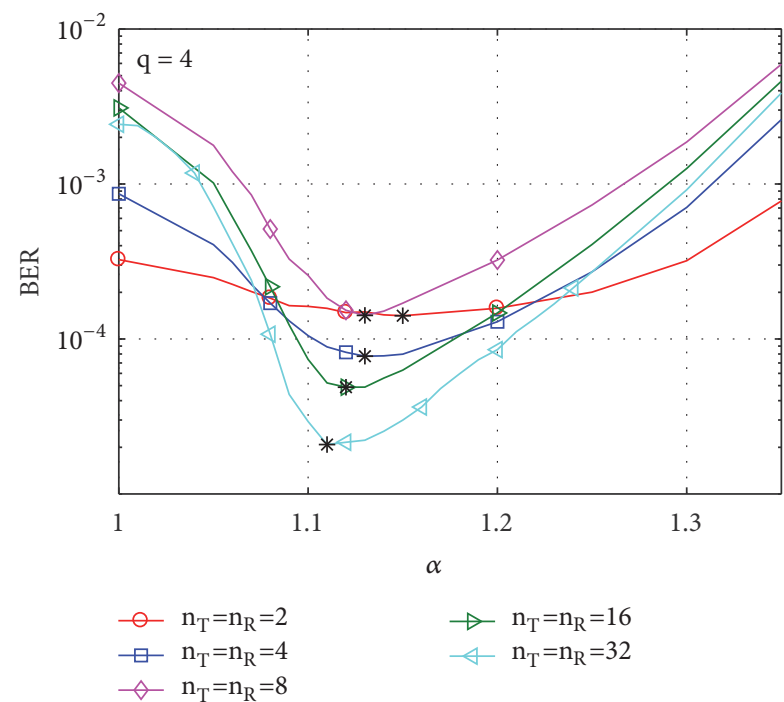

(c)

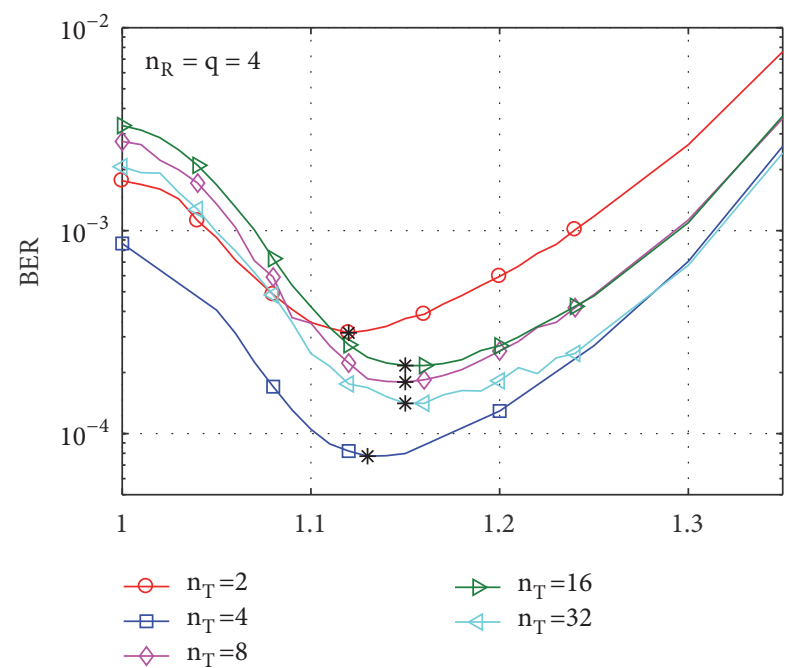

(b)

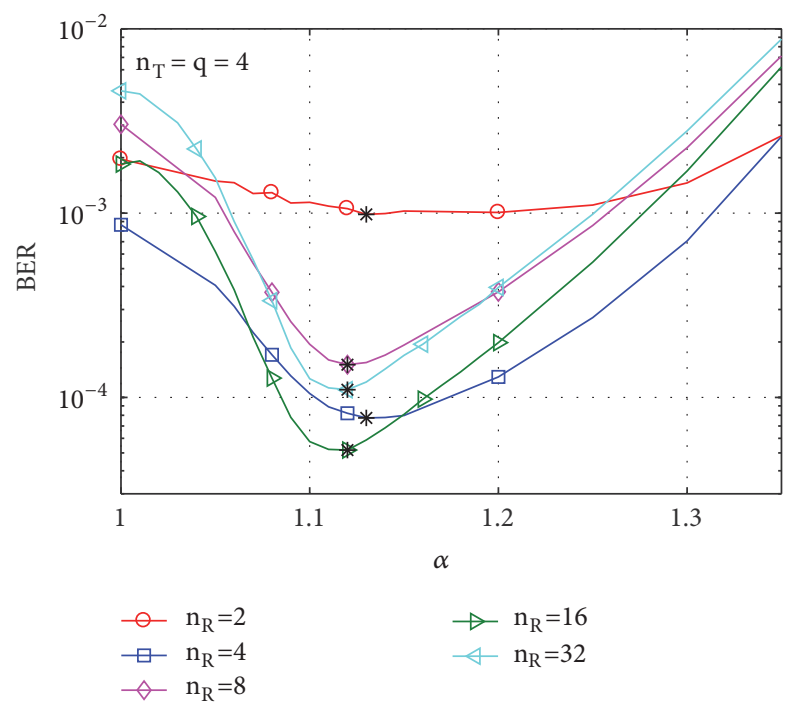

(d)

FIGURE 2: BER of the ICQSM versus $\alpha$ : (a) for $n_{T}=n_{R}=4$ and several modulation schemes, (b) for $n_{R}=4$ and several values of $n_{T}$, using 16PSK modulation, (c) for several values of $n_{T}=n_{R}$, using 16PSK modulation, and (d) for $n_{T}=4$ and several values of $n_{R}$, using 16PSK modulation.

marker in Figure 2. The results are summarized in the following:

1. Fixed $n_{T}=n_{R}=4$ and a variable $q$ : Figure $2(\mathrm{a})$ depicts the BER performance of the ICQSM versus $\alpha$ for several values of $q$. In the case of QPSK, that is, $q=2$, the optimal BER is achieved for $\alpha=\beta=1$. In the other simulated cases, the optimal $\alpha$, denoted by $\alpha_{\text {opt }}$, is inversely proportional to $q$, where it attains a minimum value of 1.04 for $q=7$. Results are summarized in Table 2(a). It will be shown in the next section that, with this very slight change in $\alpha$ from 1 to 1.04 in the case of $q=7$, a relatively large gain will be achieved due to the high symbol density in the Euclidean space

2. Fixed $n_{R}=q=4$ and a variable $n_{T}$ : Figure 2(b) depicts the BER performance of ICQSM versus $\alpha$ for several values of $n_{T}$ and a fixed $n_{R}=q=4$. The value of $\alpha_{\text {opt }}$ increases from 1.12 for $n_{T}=2$ to 1.15 for $n_{T}=8$ and keeps a fixed optimal value up to $n_{T}=32$. The full list of values is shown in the third column of Table 2(b)

3. Fixed $n_{T}=q=4$ and a variable $n_{R}$ : Figure $2(\mathrm{~d})$ depicts the BER performance of ICQSM versus $\alpha$ for several values of $n_{R}$. As the value of $n_{R}$ increases, $\alpha_{\text {opt }}$ decreases to the same optimal value of 1.12 for $n_{R}=8,16$ and 32 . These values are summarized in Table 2(d)

4. Fixed $q=4$ and a variable $n_{T}=n_{R}$ : Figure 2(c) depicts the BER performance of ICQSM versus $\alpha$ for several values of $n_{T}=n_{R}$. The optimal value of $\alpha$ inversely proportional to $n_{T}=n_{R}$ with maximum and minimum values of 1.15 and 1.11 for $n_{T}=n_{R}=2$ 


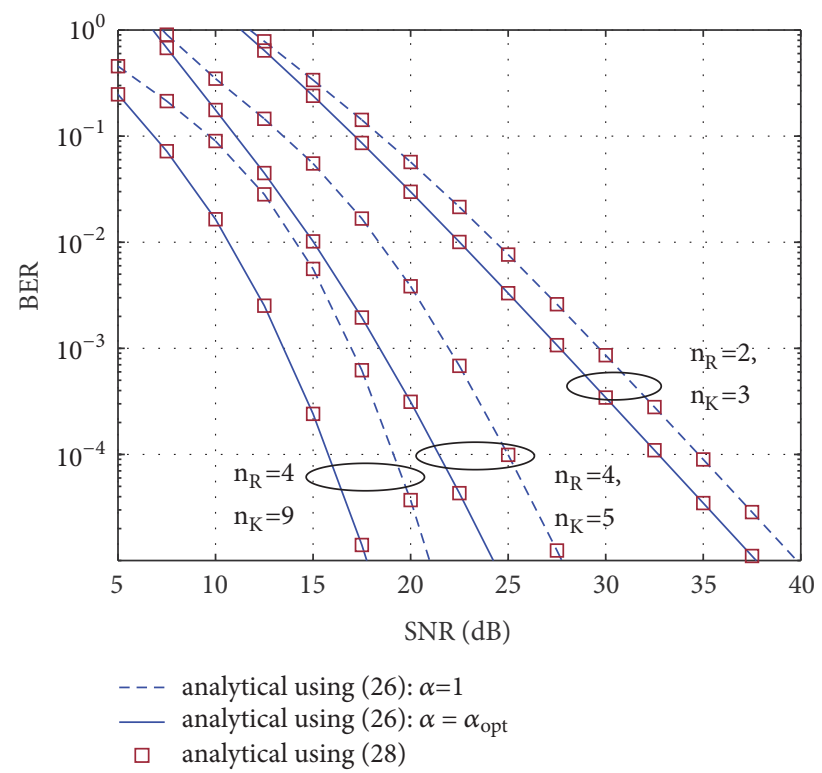

FIGURE 3: Analytical BER of the ICQSM using (26) and (28).

and $n_{T}=n_{R}=32$, respectively. Based on the earlier discussion, it stems out that while the values of both $n_{T}$ and $n_{R}$ are equal, the trend of $\alpha_{\text {opt }}$ follows that depicted in Figure 2(d). This implies that $n_{R}$ has a more dominant effect on the value of $\alpha_{\text {opt }}$ as compared to $n_{T}$

Excluding the cases when $n_{T}$ or $n_{R}=2$, a single average value of $\alpha_{\mathrm{opt}}$ can be used while marginally affecting the achieved BER. For instance, Figure 2(d) shows that if an average value of $\bar{\alpha}=(1.13+1.12+1.12+1.12) / 4=1.1225$ is used for $n_{R} \leq 4$, the BER will be almost intact. This reduces the computational cost of the offline optimization.

\section{Simulation Results}

In this section, we assume that only the receiver has full knowledge of the channel state information. The optimal rotation angle, as was derived analytically, is equal to $\pi / M$ for an M-ary PSK modulation scheme.

The analytical results of (28) are first verified in Figure 3 for several scenarios, using 16PSK modulation sets for $\Omega_{a}$ and $\Omega_{b}$ and the optimal rotation angle. The dashed and solid lines represent the analytical average BER of ICQSM for $\alpha=1$ and the optimal $\alpha$, respectively, obtained through substituting (26) in (23) and (24). The square markers indicate the average BER obtained by substituting (28) in (23) and (24). It is evident that both results coincide and therefore the analytical derivation in (28) is accurate.

Figure 4 depicts the performance of the ICQSM with the proposed modulation sets for a fixed $n_{R}=n_{T}=4$ and several modulation schemes. The results are depicted for the optimal radii and equal radii of $\alpha=\beta=1$. At a target BER of $10^{-4}$, the ICQSM with the proposed constellation design outperforms the conventional ICQSM by $1.4,3,4$, and $4.5 \mathrm{~dB}$ for $q=3,4,5$, 6 , and 7 , respectively. This relatively high gain is achieved at

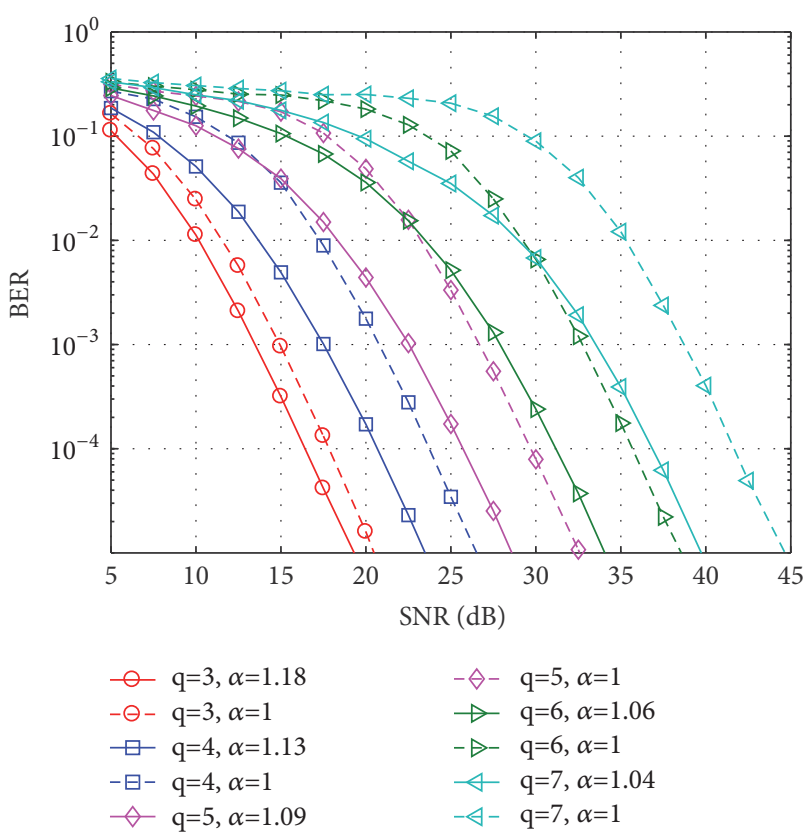

FIGURE 4: BER of the ICQSM with the proposed modulation sets versus that of the conventional ICQSM, for a fixed $n_{R}=n_{T}=4$ and several values of $q$.

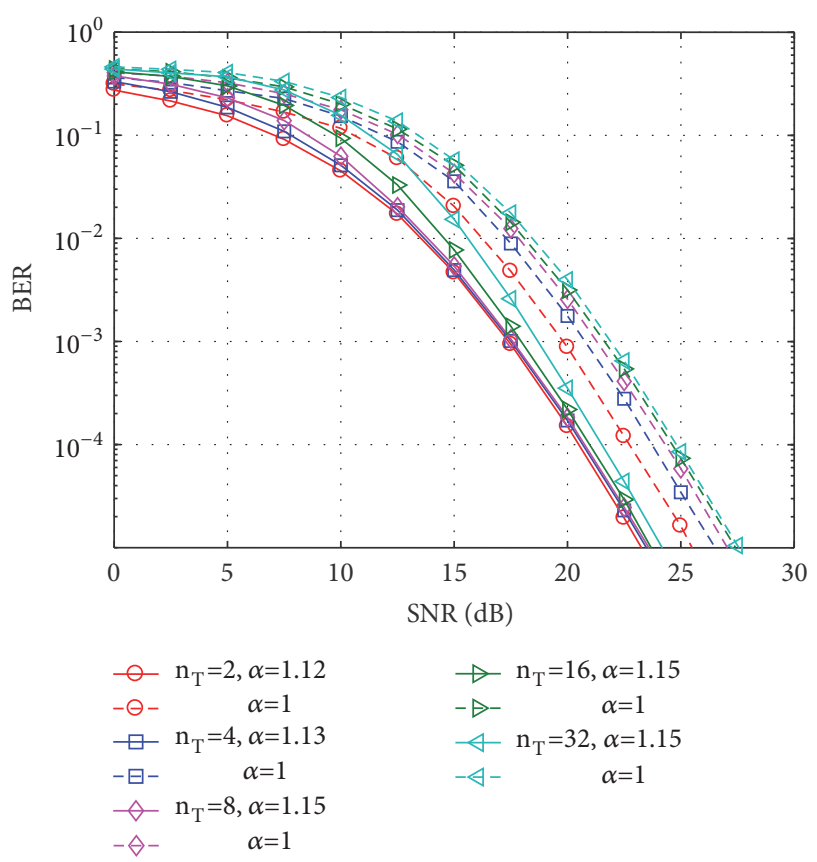

FIGURE 5: BER of ICQSM with the proposed modulation sets versus that of the conventional ICQSM, for a fixed $n_{R}=4$ and several values of $n_{T}$, using 16PSK modulation.

no cost as the parameters of constellation sets are optimized analytically, in the case of $\theta$, and independent of the channel realization $\mathbf{H}$ in the case of $\alpha$ and $\beta$.

Figure 5 depicts the performance of the ICQSM with the proposed modulation sets for a fixed $n_{R}=q=4$ and several values of $n_{T}$. The optimal values of $\alpha$ are indicated in 


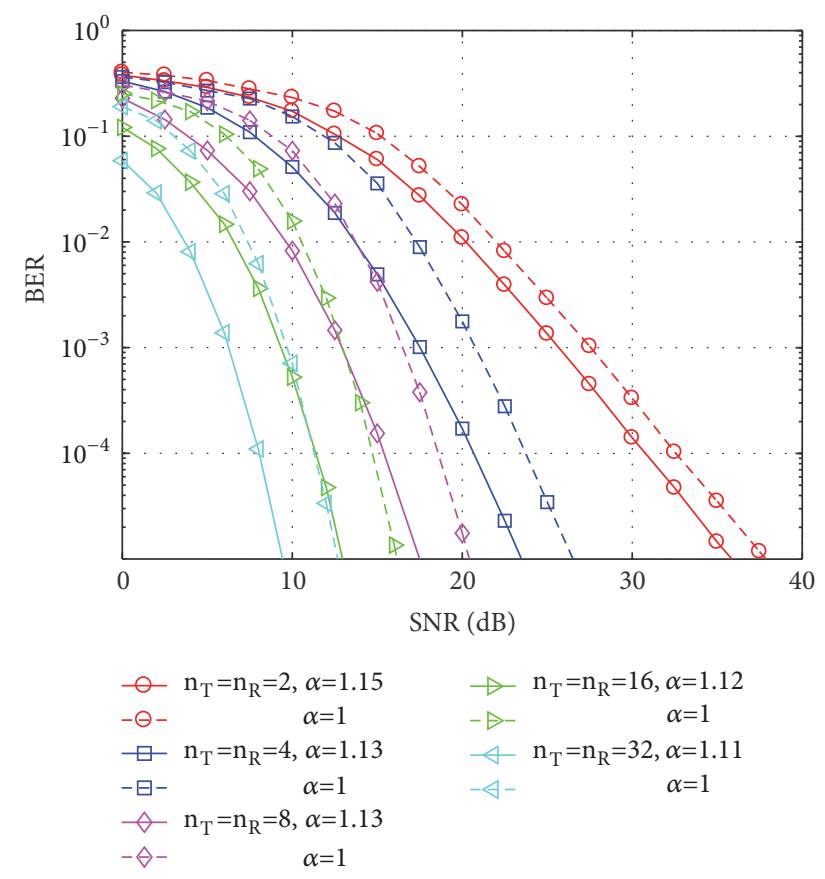

FIGURE 6: BER of ICQSM with the proposed modulation sets versus that of the conventional ICQSM, for several $n_{T}=n_{R}$ values, using 16PSK modulation.

the legend for each simulated scenario. A gain of 2.2, 3, 3.6, 3.6 , and $3.3 \mathrm{~dB}$ is achieved using $2,4,8,16$, and 32 transmit antennas, respectively.

The simulation results for the ICQSM system for several $n_{T}=n_{R}$ using 16PSK are depicted in Figure 6. At a target BER of $10^{-4}$, the ICQSM with optimal parameters achieves $1.8,3.1,3.2,3.3$, and $3.2 \mathrm{~dB}$ for $n_{R}=n_{T}=2,4,8,16$, and 32, respectively.

The BER of the ICQSM with the improved modulation sets for a fixed $n_{T}$ of four and several values of $n_{R}$ is depicted in Figure 7. At a target BER of $10^{-4}$, a gain in SNR of 1.6, 3.1, $2.8,2.8$, and $2.9 \mathrm{~dB}$ is achieved for $n_{R}=2,4,8,16$, and 32, respectively. Also, the average value of the optimal $\alpha$, given by $\bar{\alpha}=(1.13+1.12+1.12+1.12) / 4=1.1225$, is used as a single optimal value for $n_{R} \geq 4$ to generate the dash-dot curves in Figure 7. The degradation in the average BER is unremarkable. This reduces the computational complexity of obtaining the optimal modulation sets, where a single set of values is obtained for a wide range of system configurations. Finally, Figure 8 depicts the performance of the ICQSM with the improved constellation sets for a fixed $n_{T}=4$ and several values of $n_{R}$, using 32PSK. An equal gain of about $3.4 \mathrm{~dB}$ is achieved assuming $n_{R}=8$ and $n_{R}=16$ at a target BER of $10^{-4}$. A gain of about $2.6 \mathrm{~dB}$ is achieved at a target BER of $10^{-2}$, using $n_{R}=2$.

Several future works building on CQSM/ICQSM and the constellation set design are possible. For instance, authors will investigate the design of a generalized CQSM, where each signal symbol is transmitted from a subset of antennas. The modulation sets will be then redesigned for the generalized schemes.

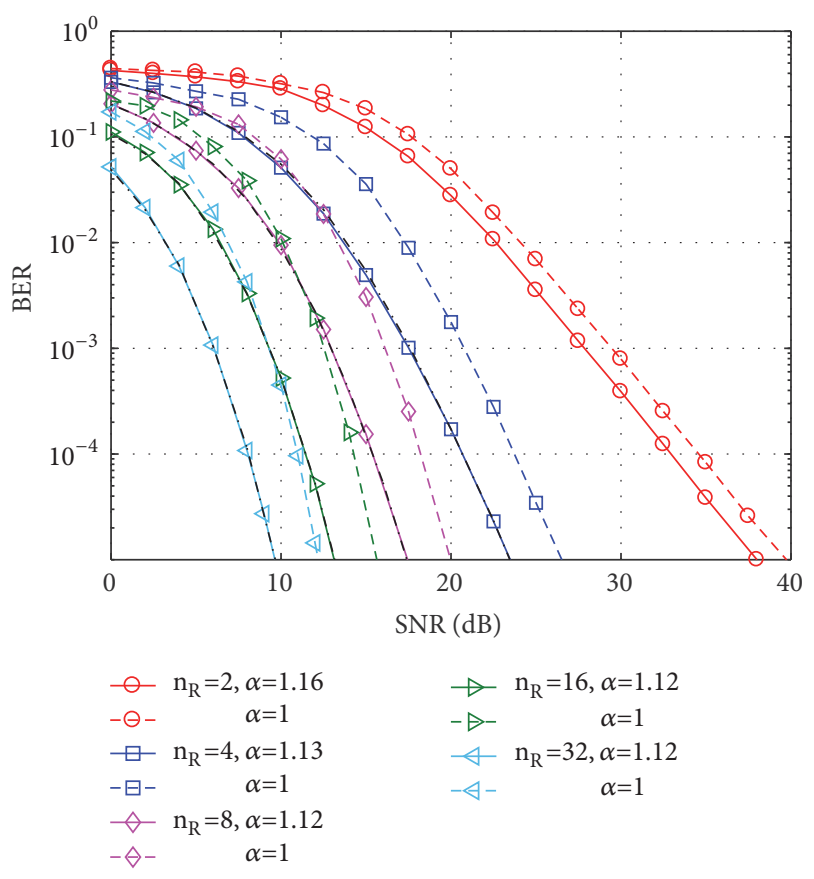

FIGURE 7: BER of ICQSM with the proposed modulation sets versus that of the conventional ICQSM, for a fixed $n_{T}=4$ and several values of $n_{R}$, using 16PSK modulation.

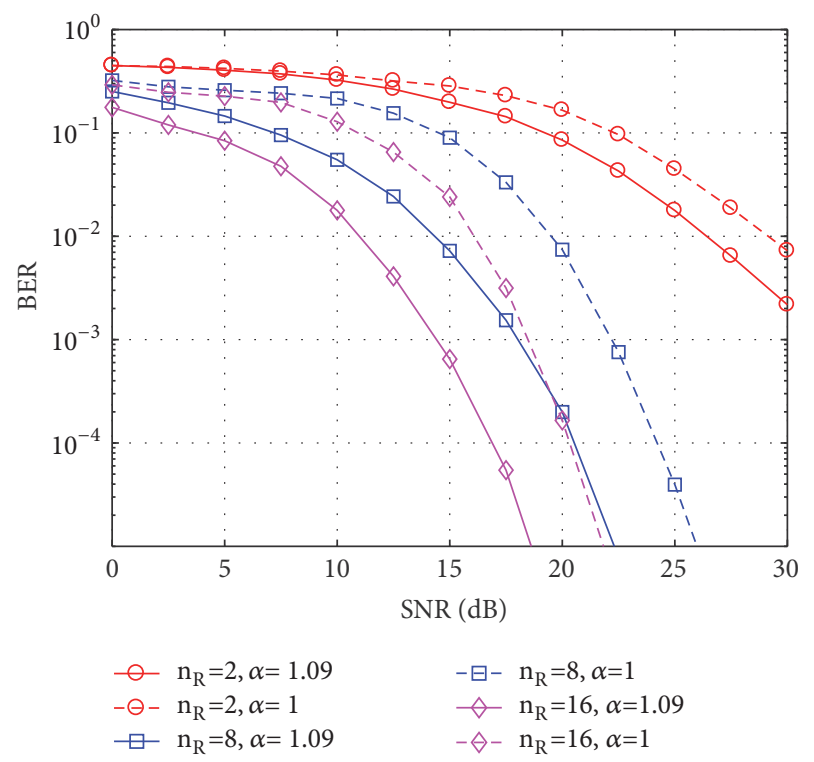

FIGURE 8: BER of ICQSM with the proposed modulation sets versus that of the conventional ICQSM, for a fixed $n_{T}=4$ and several values of $n_{R}$, using 32PSK modulation.

\section{Conclusions}

Two exhaustive search methods of the optimal modulation sets for the ICQSM system are investigated. After showing that their computational complexity render the system unpractical, we introduce a low-complexity method that reduces the average unconditional error (AUE). We first 
show that the AUP depends on the power of signal symbols, the Euclidean distance between the symbols of each set, and the Euclidean distance between the symbols within the two sets. The optimization reduces, therefore, to finding the optimal rotation angle of the second modulation set and the optimal radius of each of the modulation sets. We analytically formulate the optimal rotation angle for any modulation order and then obtain the optimal radii for a wide range of system configurations. The simulation results show that the gain of the proposed modulation sets exceeds $4 \mathrm{~dB}$ for high order modulation.

\section{Data Availability}

The data used to support the findings of this study are available from the corresponding author upon request.

\section{Conflicts of Interest}

The authors declare that they have no conflicts of interest.

\section{Authors' Contributions}

The first and second authors have contributed equally to this paper.

\section{Acknowledgments}

The second author was supported by research stipend by KOREATECH in the period of 2017-2018. The first and third authors are supported by a grant from National Research Foundation of Korea funded by the Korean Government (Ministry of Science, ICT and Future Planning) (NRF2017R1A2B2012337).

\section{References}

[1] E. Basar, M. Wen, R. Mesleh, M. Di Renzo, Y. Xiao, and H. Haas, "Index Modulation Techniques for Next-Generation Wireless Networks," IEEE Access, vol. 5, pp. 16693-16746, 2017.

[2] E. g. Basar, U. Aygolu, E. Panayirci, and H. V. Poor, "Orthogonal frequency division multiplexing with index modulation," IEEE Transactions on Signal Processing, vol. 61, no. 22, pp. 5536-5549, 2013.

[3] G. Kaddoum, M. F. A. Ahmed, and Y. Nijsure, "Code index modulation: A high data rate and energy efficient communication system," IEEE Communications Letters, vol. 19, no. 2, pp. 175-178, 2015.

[4] J. Zhang, Y. Wang, J. Zhang, and L. Ding, "Polarization shift keying (PolarSK): System scheme and performance analysis," IEEE Transactions on Vehicular Technology, vol. 66, no. 11, pp. 10139-10155, 2017.

[5] M. Wen, E. Basar, Q. Li, B. Zheng, and M. Zhang, "MultipleMode Orthogonal Frequency Division Multiplexing with Index Modulation," IEEE Transactions on Communications, vol. 65, no. 9, pp. 3892-3906, 2017.

[6] S. Althunibat and R. Mesleh, "Index Modulation for Clusterbased Wireless Sensor Networks," IEEE Transactions on Vehicular Technology, pp. 1-1, 2018.
[7] E. Basar, "Index modulation techniques for 5G wireless networks," IEEE Communications Magazine, vol. 54, no. 7, pp. 168175,2016

[8] M. Wen, X. Cheng, and L. Yang, Index Modulation for 5G Wireless Communications, Springer, 2017.

[9] R. Y. Mesleh, H. Haas, S. Sinanović, C. W. Ahn, and S. Yun, "Spatial modulation," IEEE Transactions on Vehicular Technology, vol. 57, no. 4, pp. 2228-2241, 2008.

[10] A. Younis, R. Mesleh, and M. Di Renzo, "Generalized spatial modulation for large-scale MIMO, in," in Proc. Eusipco, pp. 346-350, Portugal, Lisbon, September 2014.

[11] L. Yang, "Transmitter Preprocessing Aided Spatial Modulation for Multiple-Input Multiple-Output Systems," in Proceedings of the 2011 IEEE Vehicular Technology Conference (VTC 2011Spring), pp. 1-5, Budapest, Hungary, May 2011.

[12] R. Zhang, L.-L. Yang, and L. Hanzo, "Generalised pre-coding aided spatial modulation," IEEE Transactions on Wireless Communications, vol. 12, no. 11, pp. 5434-5443, 2013.

[13] M. Mohaisen and K. Chang, "On the achievable improvement by the linear minimum mean square error detector," in Proceedings of the 2009 9th International Symposium on Communications and Information Technology (ISCIT), pp. 770774, Icheon, South Korea, September 2009.

[14] M. Mohaisen and V. Pruks, "Downlink macro-diversity precoding-aided spatial modulation, Journal of Communications and Networks," 2017.

[15] R. Mesleh, S. S. Ikki, and H. M. Aggoune, "Quadrature spatial modulation," IEEE Transactions on Vehicular Technology, vol. 64, no. 6, pp. 2738-2742, 2015.

[16] R. Mesleh, S. Althunibat, and A. Younis, "Differential Quadrature Spatial Modulation," IEEE Transactions on Communications, vol. 65, no. 9, pp. 3810-3817, 2017.

[17] M. Mohaisen and S. Lee, "Complex quadrature spatial modulation," ETRI Journal, vol. 39, no. 4, pp. 514-524, 2017.

[18] M. Mohaisen, "Increasing the minimum Euclidean distance of the complex quadrature spatial modulation," IET Communications, vol. 12, no. 7, pp. 854-860, 2018.

[19] S. Althunibat and R. Mesleh, "A Bit-To-Symbol Mapping Scheme for Spatial Modulation with Partial Channel State Information," IEEE Communications Letters, vol. 21, no. 5, pp. 995-998, 2017.

[20] P. Yang, Y. Xiao, L. Yin, Q. Tang, S. Li, and L. Hanzo, "Hybrid Bitto-Symbol Mapping for Spatial Modulation," IEEE Transactions on Vehicular Technology, vol. 65, no. 7, pp. 5804-5810, 2016.

[21] N. Ayyad, N. Alqaramseh, M. Qazzaz, S. Althunibat, and W. A. Shehab, "Setup optimization in spatial modulation systems: A simulation study," in Proceedings of the 2017 8th International Conference on Information and Communication Systems (ICICS), pp. 241-245, Irbid, Jordan, April 2017.

[22] M. Maleki, H. R. Bahrami, S. Beygi, M. Kafashan, and N. H. Tran, "Space modulation with CSI: Constellation design and performance evaluation," IEEE Transactions on Vehicular Technology, vol. 62, no. 4, pp. 1623-1634, 2013.

[23] M. D. Renzo, H. Haas, A. Ghrayeb, S. Sugiura, and L. Hanzo, "Spatial modulation for generalized MIMO: challenges, opportunities, and implementation," Proceedings of the IEEE, vol. 102, no. 1, pp. 56-103, 2014.

[24] M. K. Simon and M. Alouini, Digital Communication over Fading Channels, John Wiley \& Sons, Inc., Hoboken, NJ, USA, 2004. 
[25] M. Di Renzo and H. Haas, "Bit error probability of SMMIMO over generalized fading channels," IEEE Transactions on Vehicular Technology, vol. 61, no. 3, pp. 1124-1144, 2012.

[26] P. Yang, Y. L. Guan, Y. Xiao, M. Di Renzo, S. Li, and L. Hanzo, "Transmit precoded spatial modulation: maximizing the minimum euclidean distance versus minimizing the bit error ratio," IEEE Transactions on Wireless Communications, vol. 15, no. 3, pp. 2054-2068, 2016.

[27] Q. Shi and Y. Karasawa, "An accurate and efficient approximation to the gaussian Q-function and its applications in performance analysis in nakagami-m fading," IEEE Communications Letters, vol. 15, no. 5, pp. 479-481, 2011.

[28] M. López-Benítez and F. Casadevall, "Versatile, accurate, and analytically tractable approximation for the Gaussian Qfunction," IEEE Transactions on Communications, vol. 59, no. 4, pp. 917-922, 2011.

[29] R. P. Brent, "Multiple-precision zero-finding methods and the complexity of elementary function evaluation," Analytic computational complexity (Proc. Sympos., Carnegie-Mellon Univ., PITtsburgh, PA., 1975), Academic Press, New York, pp. 151-176, 1976. 


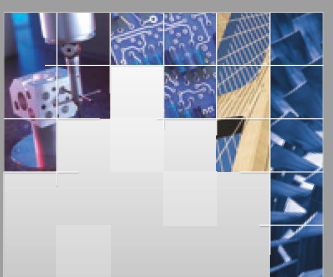

\section{Enfincering}
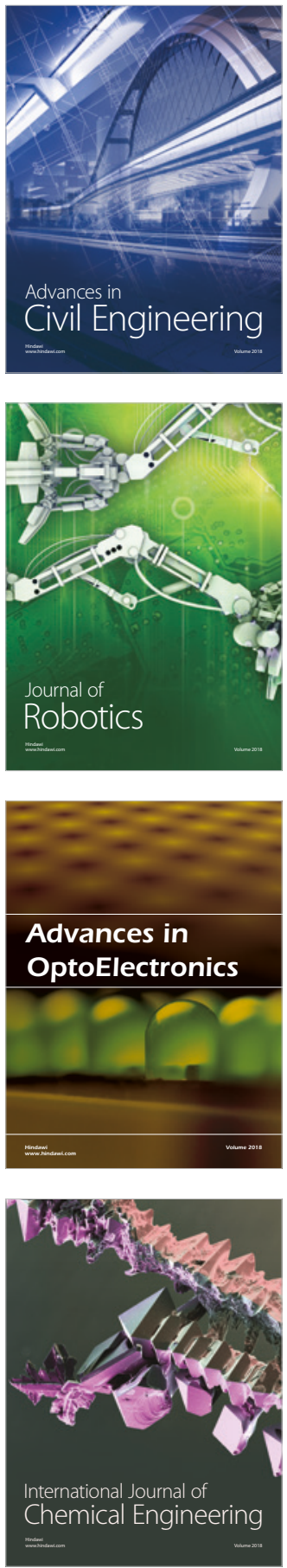

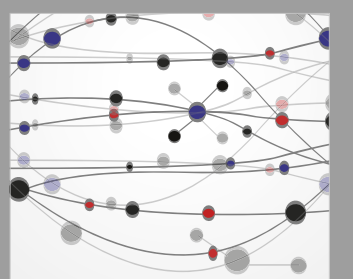

\section{Rotating \\ Machinery}

The Scientific World Journal

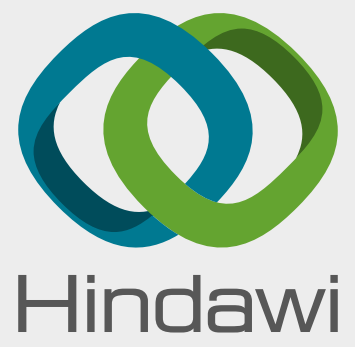

Submit your manuscripts at

www.hindawi.com
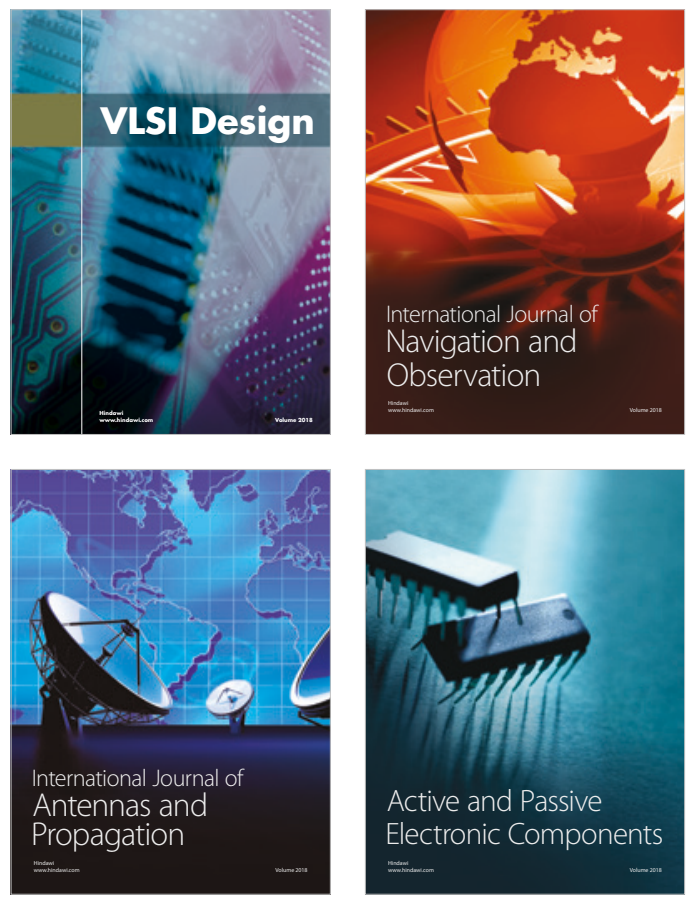
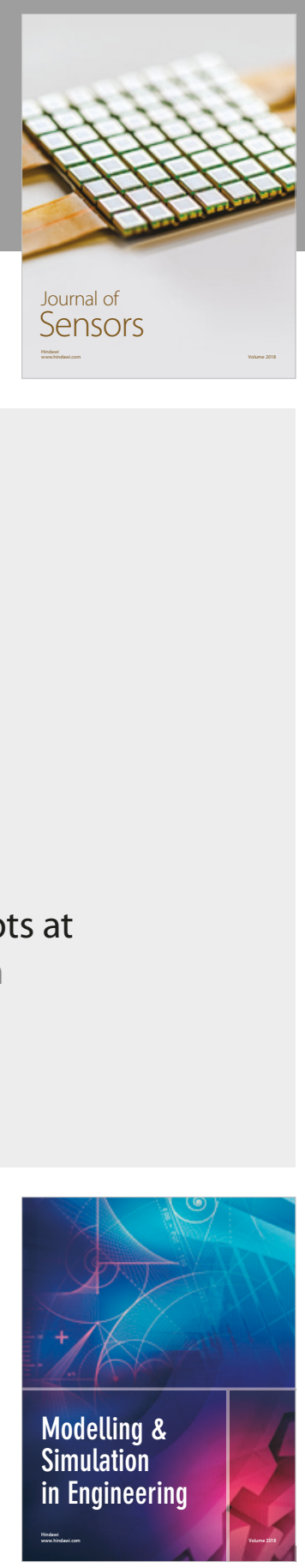

\section{Advances \\ Multimedia}
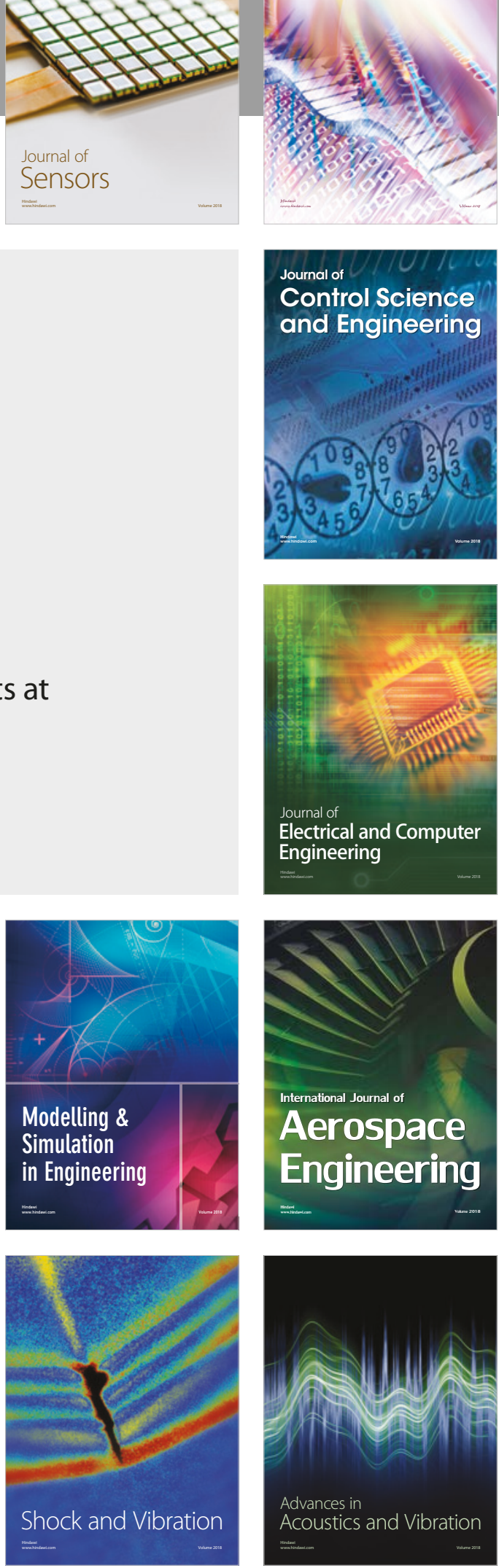